\begin{tabular}{|c|l|}
\hline Title & Measurement technique for pumping performance of a centrifugal collector under microgravity \\
\hline Author(s) & Totani, T suy oshi; Itami, Masahiko; Nagata, Harunori; Kudo, Isao; I wasaki, A kira \\
\hline Citation & $\begin{array}{l}\text { Review of Scientific Instruments, 75(2), 515-523 } \\
\text { https://doi.org/10.1063/1.1638872 }\end{array}$ \\
\hline Issue Date & 200402 \\
\hline Doc URL & http://hdl.handle.net/2115/5866 \\
\hline Rights & Copyright $\odot 2004$ A merican Institute of Physics \\
\hline Type & article \\
\hline File Information & RSI 75-2.pdf \\
\hline
\end{tabular}

Instructions for use 


\title{
Measurement technique for pumping performance of a centrifugal collector under microgravity
}

\author{
Tsuyoshi Totani ${ }^{\mathrm{a})}$ \\ Division of Mechanical Science, Hokkaido University, Kita 13 Nishi 8, Kita-ku, Sapporo, \\ Hokkaido 060-8628, Japan \\ Masahiko Itami \\ Matsushita Electric Industrial Co., Ltd., Home Appliance \& Housing Electronics Company, \\ 1-2 Kamisu-cho, Toyonaka, Osaka 561-0823, Japan \\ Harunori Nagata and Isao Kudo \\ Division of Mechanical Science, Hokkaido University, Kita 13 Nishi 8, Kita-ku, Sapporo, \\ Hokkaido 060-8628, Japan \\ Akira Iwasaki \\ National Institute of Advanced Industrial Science and Technology, Tsukuba Central 2, Tsukuba, \\ Ibaraki 305-8568, Japan
}

(Received 3 July 2003; accepted 6 November 2003)

\begin{abstract}
A measurement technique for obtaining the pumping performance of a centrifugal collector under microgravity has been developed and evaluated through microgravity experiments. These tests have been conducted under conditions such that the pressure sensors cannot easily detect the pressure rise of the liquid working fluid. These conditions have a pressure increase smaller than $400 \mathrm{~Pa}$. The characteristic of the head produced in a centrifugal collector calculated from experimental data agrees well with that predicted theoretically from the velocity and the pressure generated by rotation of the centrifugal collector. It is concluded from this result that the measurement technique can correctly obtain the pumping performance of the centrifugal collector under microgravity. The centrifugal collector has produced the head of $0.041 \mathrm{~m}$ at the rotation speed of $223 \mathrm{rpm}$ under microgravity. The working fluid is silicon oil. This head corresponds to the pressure rise of approximately $390 \mathrm{~Pa}$. (C) 2004 American Institute of Physics. [DOI: 10.1063/1.1638872]
\end{abstract}

\section{INTRODUCTION}

Disposing of large quantities of waste heat is one of the technical issues to be considered in the realization of large structures in space which handle high power (from megawatts to gigawatts), such as solar power satellites, space factories in orbit and bases on the moon. The liquid droplet radiator (LDR) is an important candidate for resolving this issue. Its lightweight structure, strong resistance to meteorite impacts, small storage volume requirement at launch and easy deployment in space make it a very attractive heat rejection system for the above applications. The operation of the LDR is schematically shown in Fig. 1. The LDR consists of a droplet generator, a droplet collector, a circulating pump, and a heat exchanger. Working fluid is heated through a heat exchanger by waste heat generated in a large structure in space. The working fluid is emitted into space as multiple streams of liquid droplets through nozzles of the droplet generator, toward a droplet collector. During the flight from the droplet generator to the droplet collector in space, the droplets lose thermal energy by radiative heat transfer. After the cooled droplets are captured by the droplet collector, the working fluid is recycled to the heat exchanger by a circulating pump. Liquids with low vapor pressure are candidates for the working fluid from the viewpoint of minimizing

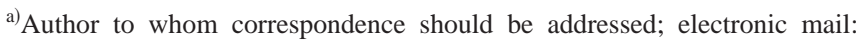
tota@eng.hokudai.ac.jp
}

evaporation loss. These include silicon oils in the temperature range of $250-350 \mathrm{~K}$, tin-lead-bismuth eutectics at $400-500 \mathrm{~K}$ and liquid tin at 500-1000 K. ${ }^{1}$ Since LDRs do not require solid bodies to protect the radiating surface from punctures by small particles such as debris or small meteorites, they are lightweight, and can be stowed in a small volume at launch and easily deployed in orbit. ${ }^{2}$ Massardo et al. have reported that the specific mass of a solar power dynamic system with an LDR is $27 \%$ smaller than that of a system with a conventional heat pipe radiator. ${ }^{3}$

Droplet collectors for LDRs should have two functions. One is to capture incident droplets without splashing and separation of the working fluid because these lead to the loss of working fluid in space. The other is to pump the working fluid into the circulating pump, which is set downstream of a droplet collector, in order to establish fluid circulation. Since droplet collectors operate in the high vacuum environment of space, there is no static head exerted on the working fluid in the collectors. Although a number of collector concepts have been proposed, those that have been investigated in detail are linear collectors and centrifugal collectors. ${ }^{4-8}$ They are respectively shown in Figs. 2 and 3. The former collector captures incident droplets and transports the captured droplets to the circulating pump by utilizing an auxiliary liquid film on its internal surface. The latter collector captures incident droplets on a liquid film inside a spinning cone. The liquid film is formed through the migration of the incident droplets radially outward due to the centrifugal force. The liquid 


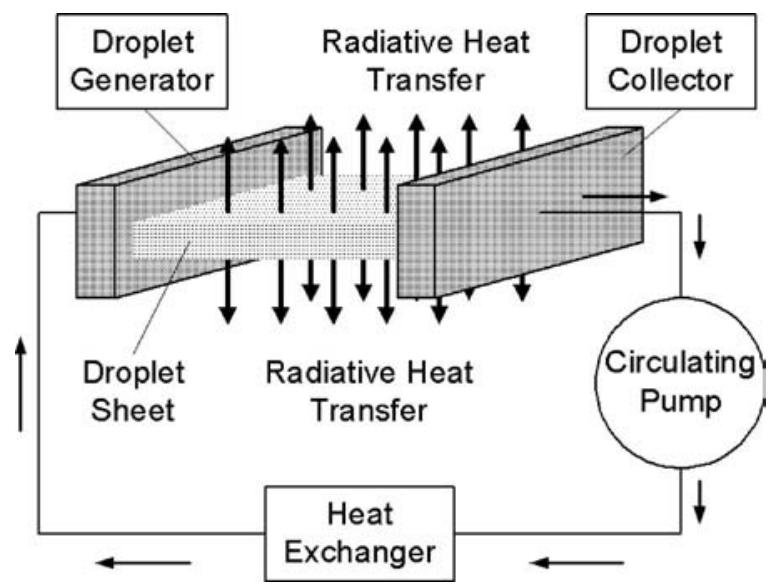

FIG. 1. Schematic diagram of liquid droplet radiator.

flows out through stationary pitot tubes that are immersed in the rotating liquid pool. The latter collectors have the advantage that the liquid films of these collectors splash much less than those of the former collectors because of the centrifugal force acting on the liquid films. We consider that centrifugal collectors are more appropriate for the droplet collector than the linear collectors.

A centrifugal collector with a pitot tube for fluid recovery such as that shown in Fig. 3 was developed and produced the head of $34 \mathrm{kPa}$ at $300 \mathrm{rpm}$ under normal gravity. ${ }^{4} \mathrm{Few}$ reports have described the performance of centrifugal droplet collectors under microgravity. The surface position of the working fluid in centrifugal collectors under microgravity is different from that under normal gravity, as shown in Fig. 4. Since the pressure at the entrance of the pitot tube under microgravity differs from that under normal gravity, the pumping performance under microgravity is not equal to that under normal gravity. The most cost-effective and easily utilized microgravity platforms are drop shafts or drop towers. The performance tests of a droplet generator, ${ }^{9}$ a linear droplet collector, ${ }^{10}$ and a gear pump ${ }^{11}$ have been conducted at the drop shafts of the Micro-Gravity Laboratory of Japan Inc. (MGLAB $)^{12,13}$ and the Japan Microgravity Center Inc. (JAMIC) ${ }^{14}$ The head, which is an index of pumping performance, is most simply measured under normal gravity as the height of the level of the pumped-up liquid from a reference level. However, this measurement method is ineffective under microgravity. The head can also be calculated from the velocities and the pressures at the upstream and at the down-

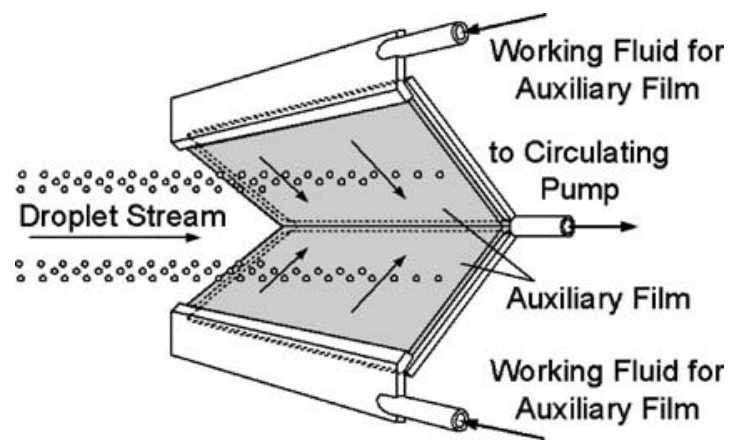

FIG. 2. Concept of linear collector.

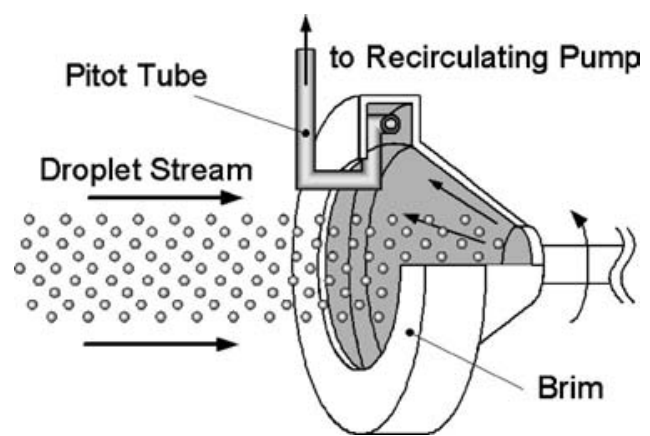

FIG. 3. Concept of centrifugal collector.

stream of pumping equipment. However, there is a size limit to the experimental setup for microgravity experiments using the drop shaft or drop tower. Centrifugal collectors with large diameters cannot be used in these microgravity experiments. On the other hand, the pressure rises generated by small diameter centrifugal collectors are so small that the pressure sensor is not able to detect them with sufficient accuracy.

The technique developed in the present work enables us to measure the head generated in the centrifugal collector under microgravity using the drop shaft and the drop tower, by filming the movement of the working fluid in a transparent tube by means of an $8 \mathrm{~mm}$ video camera. This article describes the measurement technique for the pumping performance of a centrifugal collector under microgravity using the MGLAB drop shaft and reports the test results for the pumping performance of the centrifugal collector under microgravity. These tests have been conducted under conditions such that the pressure sensors cannot easily detect the pressure rise of the liquid working fluid. These conditions have a pressure increase smaller than $400 \mathrm{~Pa}$.

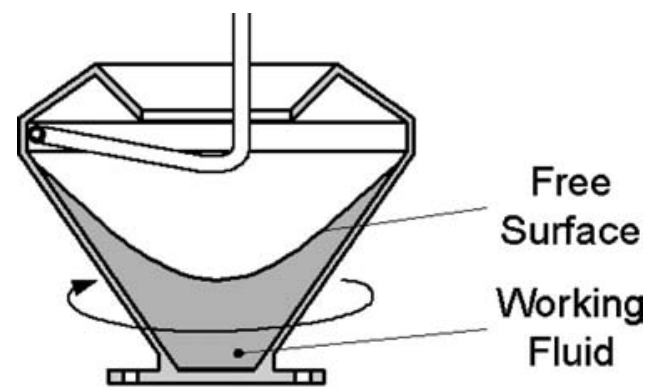

(a) Normal gravity

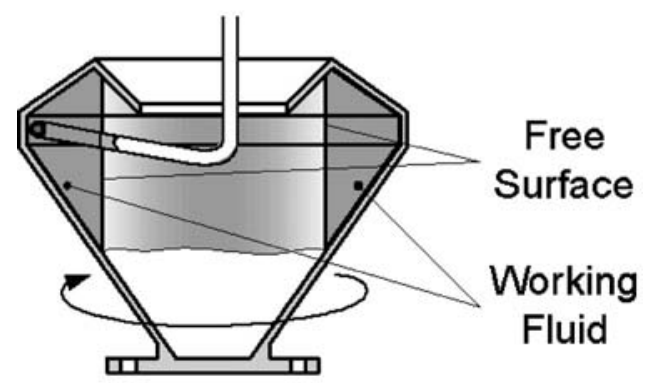

(b) Microgravity

FIG. 4. Surface positions of working fluid under normal gravity and microgravity. 


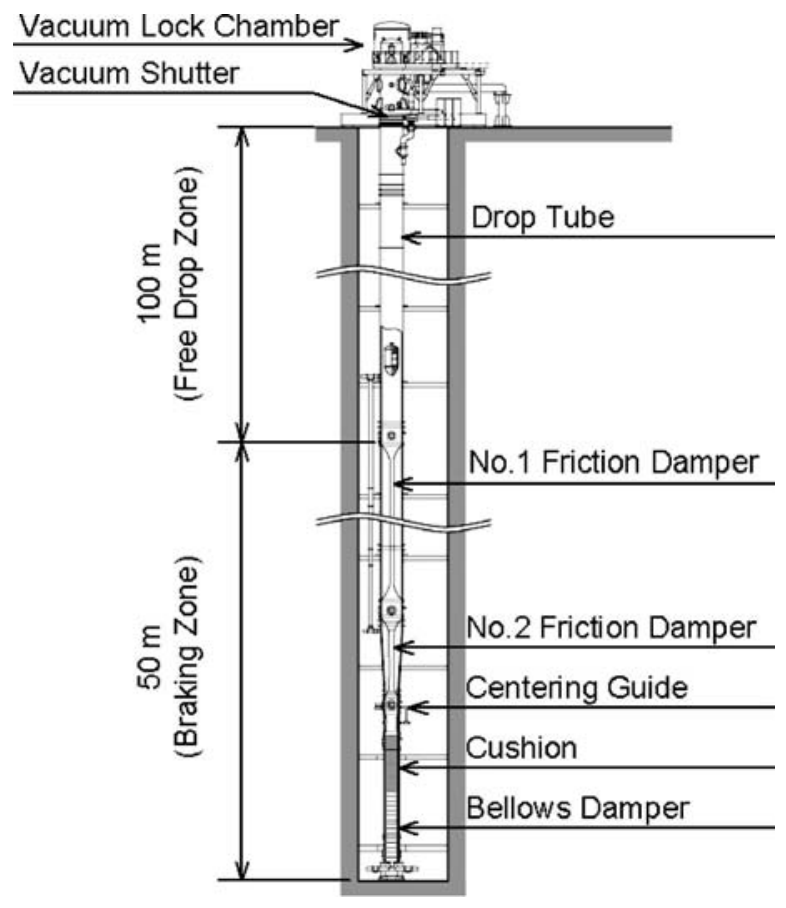

FIG. 5. Schematic drawing of MGLAB drop shaft.

\section{EXPERIMENT}

\section{A. Microgravity experiment facility ${ }^{13}$}

Figure 5 schematically shows the MGLAB drop shaft. The drop shaft is $150 \mathrm{~m}$ deep and is equipped with a drop tube of $1500 \mathrm{~mm}$ diameter. The free drop zone is $100 \mathrm{~m}$ long and the breaking zone is $50 \mathrm{~m}$ long. The free drop zone can provide the microgravity duration of $4.5 \mathrm{~s}$. The braking zone is equipped with two friction dampers and a bellows damper. These dampers are designed to maintain the deceleration of the capsule to less than $10 \mathrm{G}$ and allow the capsule to stop without damaging the capsule body, the experiment support

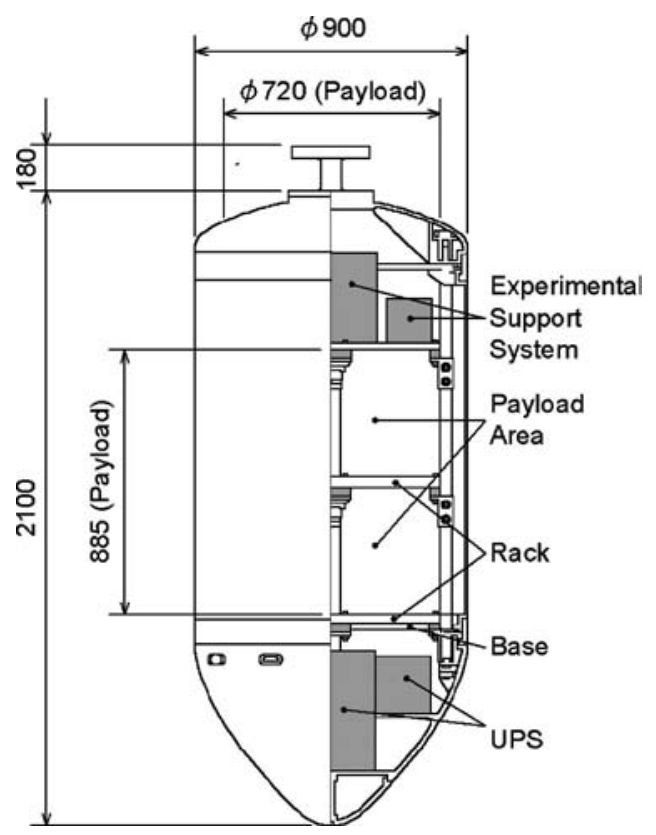

FIG. 6. Schematic diagram of drop capsule.

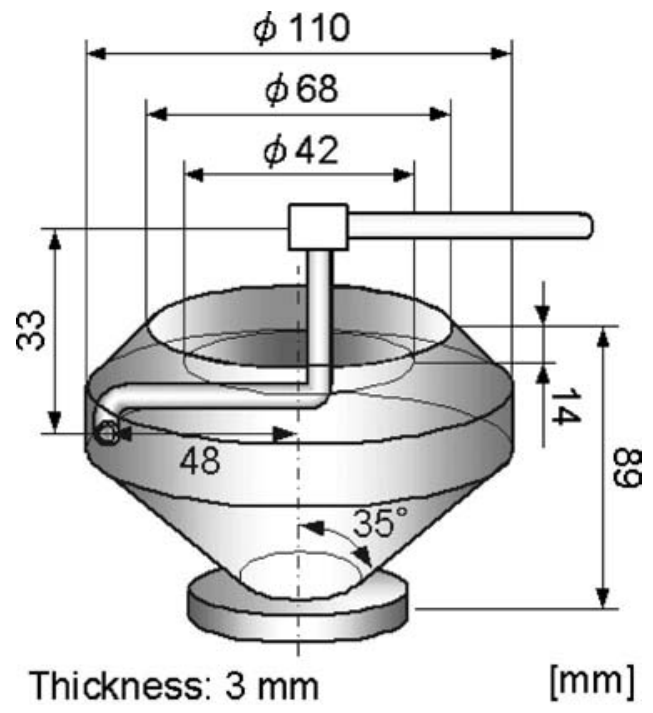

(a) Collector

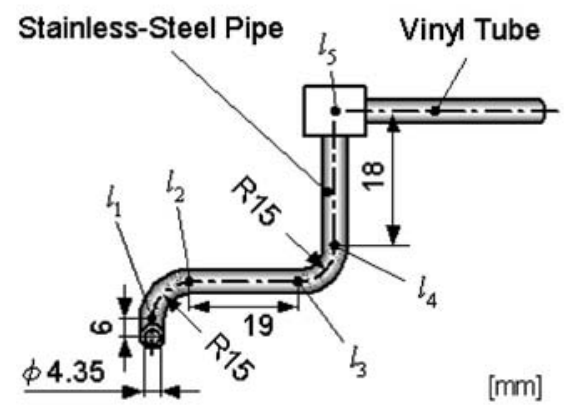

(b) Pitot tube

FIG. 7. Configuration and dimensions of centrifugal collector and pitot tube.

system, or the payload. As shown in Fig. 6, the capsule is $900 \mathrm{~mm}$ in outer diameter and $2280 \mathrm{~mm}$ in height. The payload area in the capsule where an experimental setup is mounted is $720 \mathrm{~mm}$ in diameter and $885 \mathrm{~mm}$ in height. As the figure indicates, the payload area can be separated into several stages. The maximum payload weight, including the base, the rack and balancing weights, is $400 \mathrm{~kg}$. Since the capsule is released by turning off the power supplied to the magnetic chuck used for suspending the capsule, the capsule can start to fall smoothly. The capsule falls in the drop tube which has a vacuum condition (nearly 4.0 Pa). A high-quality microgravity environment $\left(10^{-3}-10^{-5} \mathrm{G}\right)$ is supplied.

\section{B. Experimental apparatus}

Figure 7 shows the configuration and the dimensions of (a) a centrifugal collector and (b) a pitot tube used in a series of performance tests. The distance from the rotation axis to the entrance of the pitot tube is $48 \mathrm{~mm}$. The angle between the lower conical surface and the rotation axis is $35^{\circ}$. Working fluid is placed in the centrifugal collector before the capsule is assembled for the fall. As shown in Fig. 4, the quantity of working fluid put in the centrifugal collector is set such that the working fluid does not touch the tip of the pitot tube under normal gravity, and does not overflow the brim of 


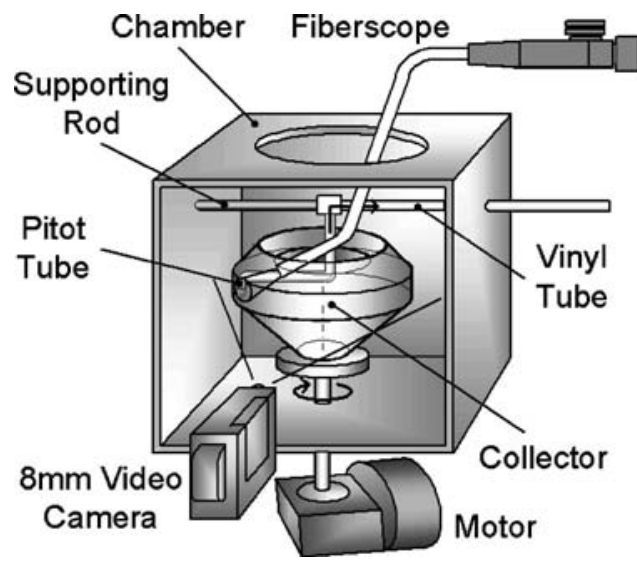

FIG. 8. Schematic illustration of experimental setup.

the centrifugal collector under microgravity at the maximum revolution of $223 \mathrm{rpm}$ in a series of performance tests. Through a series of performance tests, the quantity of working fluid has been set at $80 \mathrm{ml}$.

The experimental setup including the observation devices is schematically shown in Fig. 8. The centrifugal collector is rotated by the AC speed control motor (Oriental Motor Co., Ltd. MSD425-401). The fiberscope (Olympus Promarketing Inc. IF8C5-10) monitors the behavior of the working fluid at the tip of the pitot tube. The $8 \mathrm{~mm}$ video camera (Sony Corporation CCD-RV100) records the interface between the working fluid and the air moving in the translucent vinyl tube, and can record the movement of the working fluid at the section between 12.0 and $15.5 \mathrm{~cm}$ from the entrance of the pitot tube. An incandescent light (AC100 $\mathrm{V}, 40 \mathrm{~W}$ ) is employed for illumination. Silicon oil (Shin-Etsu Chemical Co., Ltd. KF-96 $50 \mathrm{cSt}$ ) is used as the working fluid. The properties of the working fluid are listed in Table I.

\section{Measurement method}

Figure 9 shows a schematic diagram of the rotating collector as a plan view. The centrifugal collector generates the movement of working fluid through the rotation and the pressure increase of working fluid under the centrifugal force. Applying Bernoulli's law and taking the loss of energy in the pitot tube into account, the following equation is obtained:

$$
\frac{u_{e}^{2}}{2}+\frac{p_{e}}{\rho}=\frac{u_{s}^{2}}{2}+\frac{p_{s}}{\rho}+E_{l},
$$

where $u, p$, and $\rho$ are the velocity, the gauge pressure, and the density of the working fluid, respectively. The subscripts $e$ and $s$, respectively, denote the entrance of the pitot tube and the interface between the working fluid and the atmosphere in the vinyl tube. $E_{l}$ denotes the total energy loss per unit mass from the entrance of the pitot tube to the interface in

TABLE I. Properties of working fluid.

\begin{tabular}{lcr}
\hline \hline Property & Symbol & \multicolumn{1}{c}{ Value } \\
\hline Density & $\rho$ & $957\left(\mathrm{~kg} / \mathrm{m}^{3}\right)$ \\
Coefficient of viscosity & $\mu$ & $0.0479(\mathrm{~Pa} \mathrm{~s})$ \\
Surface tension & $\sigma$ & $0.0208(\mathrm{~N} / \mathrm{m})$ \\
\hline \hline
\end{tabular}

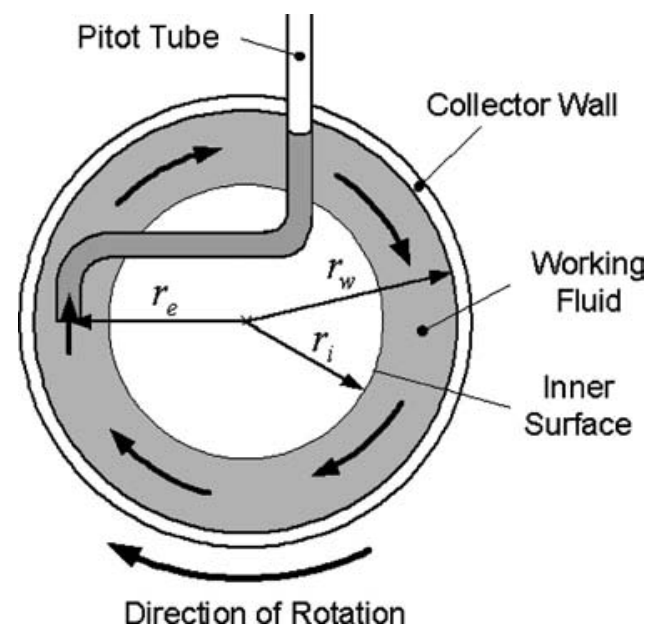

FIG. 9. Schematic diagram of centrifugal collector in plan view.

the pitot tube. The gravitational term is neglected because the head produced by the centrifugal droplet collector under microgravity is discussed. As the interface in the vinyl tube faces the atmosphere, the pressure at the surface of the working fluid $p_{s}$ is 0 . The head $H_{c}$ that the centrifugal collector generates under microgravity is given by the following equation:

$$
H_{c} \equiv \frac{1}{g}\left(\frac{u_{e}^{2}}{2}+\frac{p_{e}}{\rho}\right)=\frac{1}{g}\left(\frac{u_{s}^{2}}{2}+E_{l}\right),
$$

where $g$ is the gravitational acceleration under normal gravity.

Considering loss at the inlet of the pitot tube, pressure loss in the entrance length of the pitot tube, frictional loss in the pitot tube, and loss at the bend and elbows of the pitot tube as losses of energy, the total energy loss $E_{l}$ from the entrance of the pitot tube to the interface in the pitot tube is expressed as

$$
\begin{aligned}
& E_{l}=\zeta_{i} \frac{u_{s}^{2}}{2}+\lambda \frac{l_{s}}{d} \frac{u_{s}^{2}}{2}+E_{a} \quad\left(0<l_{s}<l_{1}\right), \\
& E_{l}=\zeta_{i} \frac{u_{s}^{2}}{2}+\lambda \frac{l_{s}}{d} \frac{u_{s}^{2}}{2}+E_{a}+\zeta_{b} \frac{l_{s}-l_{1}}{l_{2}-l_{1}} \frac{u_{s}^{2}}{2} \quad\left(l_{1}<l_{s}<l_{2}\right),
\end{aligned}
$$

$E_{l}=\zeta_{i} \frac{u_{s}^{2}}{2}+\lambda \frac{l_{s}}{d} \frac{u_{s}^{2}}{2}+E_{a}+\zeta_{b} \frac{u_{s}^{2}}{2} \quad\left(l_{2}<l_{s}<l_{3}\right)$,

$E_{l}=\zeta_{i} \frac{u_{s}^{2}}{2}+\lambda \frac{l_{s}}{d} \frac{u_{s}^{2}}{2}+E_{a}+\zeta_{b} \frac{u_{s}^{2}}{2}$

$$
+\zeta_{b} \frac{l_{s}-l_{3}}{l_{4}-l_{3}} \frac{u_{s}^{2}}{2} \quad\left(l_{3}<l_{s}<l_{4}\right),
$$

$E_{l}=\zeta_{i} \frac{u_{s}^{2}}{2}+\lambda \frac{l_{s}}{d} \frac{u_{s}^{2}}{2}+E_{a}+2 \zeta_{b} \frac{u_{s}^{2}}{2} \quad\left(l_{4}<l_{s}<l_{5}\right)$,

$E_{l}=\zeta_{i} \frac{u_{s}^{2}}{2}+\lambda \frac{l_{s}}{d} \frac{u_{s}^{2}}{2}+E_{a}+2 \zeta_{b} \frac{u_{s}^{2}}{2}+\zeta_{e} \frac{u_{s}^{2}}{2} \quad\left(l_{5}<l_{s}\right)$,

where $E_{a}$ is the pressure loss in the entrance region 


$$
\begin{aligned}
& E_{a}=\xi \frac{l_{s}}{l_{a}} \frac{u_{s}^{2}}{2} \quad\left(l_{s}<l_{a}\right), \\
& E_{a}=\xi \frac{u_{s}^{2}}{2} \quad\left(l_{a}<l_{s}\right),
\end{aligned}
$$

where $\xi$ is the coefficient of pressure loss in the entrance length $(=2.7),{ }^{15} \lambda$ is the Darcy friction factor, and $\zeta_{i}, \zeta_{b}$ and $\zeta_{e}$ are coefficients of loss at the inlet $(=0.5),{ }^{16}$ the bend, and the elbow $(=1.129)$ of the circular pipe with the smooth inner surface, respectively. ${ }^{17} d$ is the diameter of the vinyl tube $(=4.35[\mathrm{~mm}]), l_{s}$ is the distance from the entrance of the pitot tube to the interface between the working fluid and the atmosphere, $l_{1}, l_{2}, \ldots, l_{5}$ are the distances from the entrance of the pitot tube to the positions of the pitot tube shown in Fig. 7(b), respectively, and $l_{a}$ is the entrance length, which is given by ${ }^{15}$

$$
l_{a}=0.065 \operatorname{Re} d,
$$

where Re is the Reynolds number, which is defined by

$$
\operatorname{Re}=\frac{\rho u_{s} d}{\mu}
$$

In the case where the Reynolds number is sufficiently small to regard the flow in the vinyl tube as the laminar flow, the Darcy friction factor is given by ${ }^{16}$

$$
\lambda=\frac{64}{\operatorname{Re}} .
$$

The coefficient of loss at the bend is given by ${ }^{17}$

$$
\zeta_{b}=0.00515 \alpha \vartheta \operatorname{Re}^{-0.2}\left(\frac{r_{b}}{d}\right)^{0.9},
$$

where $\vartheta$ is the angle of the bend $\left(=90^{\circ}\right), r_{b}$ is the radius of curvature, and

$$
\alpha=0.95+4.42\left(\frac{r_{b}}{d}\right)^{-1.96} .
$$

Since the distance from the entrance of the pitot tube to the observational section $\left(l_{s}=0.120-0.155[\mathrm{~m}]\right)$ is longer than the entrance length $l_{a}$, the total energy loss $E_{l}$ from the entrance of the pitot tube to the observational section is represented in

$$
E_{l}=\zeta_{i} \frac{u_{s}^{2}}{2}+\xi \frac{u_{s}^{2}}{2}+\lambda \frac{l_{s}}{d} \frac{u_{s}^{2}}{2}+2 \zeta_{b} \frac{u_{s}^{2}}{2}+\zeta_{e} \frac{u_{s}^{2}}{2} .
$$

Substituting Eq. (16) into the right-hand side of Eq. (2), the following equation is obtained:

$$
H_{c}=\frac{1}{2 g}\left(1+\zeta_{i}+\xi+\lambda \frac{l_{s}}{d}+2 \zeta_{b}+\zeta_{e}\right) u_{s}^{2},
$$

where $H_{c}$ indicates the head measured in experiments. This equation indicates that the head produced in the centrifugal collector can be calculated from the velocity $u_{s}$ of the working fluid at the distance $l_{s}$ from the entrance of the pitot tube. That velocity is measured in a series of microgravity experiments using an $8 \mathrm{~mm}$ video camera, as shown in Fig. 10.

\section{Experimental procedures}

The procedure of the performance tests is described as follows. (1) Preparations for an experiment under microgravity are conducted: before the capsule is assembled, working fluid $\left(80 \mathrm{~cm}^{3}\right)$ is placed in the centrifugal collector; the rotation speed of the centrifugal collector is set; a blank videotape is inserted into the $8 \mathrm{~mm}$ video camera. (2) The $8 \mathrm{~mm}$ video camera starts to record just before the capsule begins to be assembled. The $8 \mathrm{~mm}$ video camera continues to record for about $60 \mathrm{~min}$, until the capsule is disassembled after a free fall. (3) The command for turning on the light source is transmitted from the control room at $20 \mathrm{~s}$ before a free fall. (4) The command for starting to rotate the centrifugal collector is transmitted from the control room at $7 \mathrm{~s}$ before a free fall. (5) The final drop sequence is initiated and about $2 \mathrm{~s}$ later, a gravity condition $10^{-3}$ times less than normal gravity is detected. (6) After the capsule is disassembled, the working fluid in the centrifugal collector and the pitot tube is discharged. The videotape is removed from the $8 \mathrm{~mm}$ video camera. Batteries for the $8 \mathrm{~mm}$ video camera and the fiberscope are charged.

\section{E. Method of analysis of pumping performance}

The characteristics of the head produced by the centrifugal collector are essential data for designing centrifugal collectors for practical use in space. Here, the method for deriving these characteristics from the data obtained in the microgravity experiments is described.

The streamlines of stable flow in the rotating body as shown in Fig. 9 are concentric circles on the plane vertical to the rotation axis. By applying the Navier-Stokes equations in cylindrical polar coordinates to this case, the following equations are obtained:

$$
\begin{aligned}
& \frac{u^{2}}{r}=\frac{1}{\rho} \frac{\partial p}{\partial r}, \\
& 0=\nu\left(\frac{\partial^{2} u}{\partial r^{2}}+\frac{1}{r} \frac{\partial u}{\partial r}-\frac{u}{r^{2}}\right),
\end{aligned}
$$

where $r$ is the distance from the rotation axis and $\nu$ is the kinematic viscosity $(=\mu / \rho)$. Since no slip occurs on the wall of the centrifugal collector and no tangential stresses act on the inner surface shown in Fig. 9, the boundary conditions are

$$
\begin{array}{ll}
\text { at } r=r_{w}: & u=r_{w} \omega=2 \pi r_{w} \frac{f}{60}, \\
\text { at } r=r_{i}: & \sigma_{r \theta}=\mu\left(\frac{\partial u}{\partial r}-\frac{u}{r}\right)=0,
\end{array}
$$

where $f$ is the number of rotations per minute and the subscripts $i$ and $w$ denote the inner surface of the working fluid and the wall in the centrifugal collector, respectively.

Solving Eqs. (19)-(21) for $u$, the velocity distribution is

$$
u=r \omega=2 \pi r \frac{f}{60} \text {. }
$$




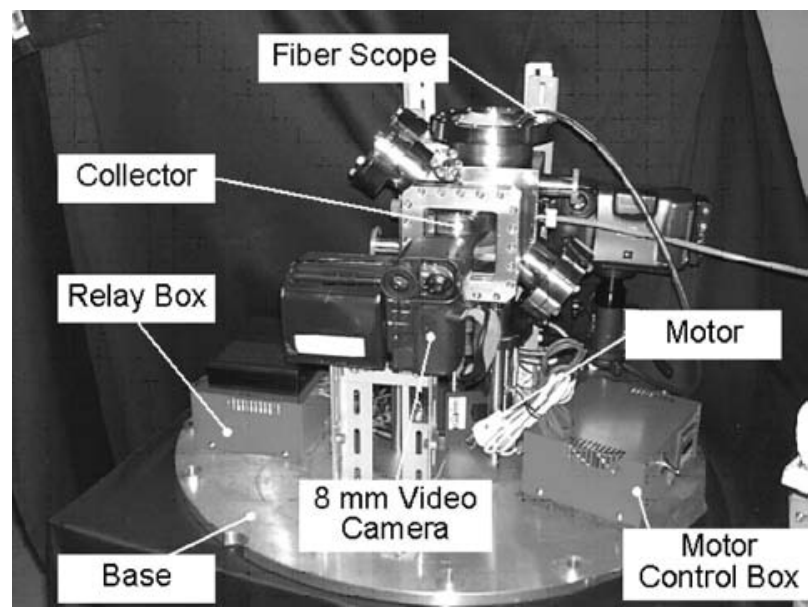

FIG. 10. Experimental setup with the base.

As shown in Fig. 9, there is a pitot tube extending into the rotating working fluid. The wake flow occurs downstream of the pitot tube. In the case of the centrifugal collectors with small circumferences, it is possible that the entrance of the pitot tube is located in the wake flow. Here, a constant $c$ is introduced as follows:

$$
u=c \cdot r \omega=c \cdot 2 \pi r \frac{f}{60} .
$$

The constant $c$ is a value specific to the shape and arrangement of the centrifugal collector and the pitot tube. It is reasonable to suppose that the constant $c$ equals 1 in the case that the circumference of the centrifugal collector is sufficiently large to recover the velocity loss in the wake flow, such as that of the centrifugal collector designed for practical use in space.

Substituting Eq. (23) into Eq. (18) and integrating from the inner surface $r_{i}$ to the entrance of the pitot tube $r_{e}$, the gauge pressure at the entrance of the pitot tube becomes

$$
p_{e}=p_{i}+\frac{1}{2} \rho\left(r_{e}^{2}-r_{i}^{2}\right)\left(2 \pi c \frac{f}{60}\right)^{2} \text {. }
$$

Since the inner surface of the working fluid faces the atmosphere, the gauge pressure at the inner surface $p_{i}$ is considered as the gauge atmospheric pressure $\left(p_{i}=0\right)$. Substituting Eq. (23) at $r=r_{e}$ and Eq. (24) into Eq. (2), the sum of the velocity head and the pressure head theoretically becomes

$$
H_{c}=\frac{1}{2 g}\left(2 r_{e}^{2}-r_{i}^{2}\right)\left(2 \pi c \frac{f}{60}\right)^{2} .
$$

As Eq. (25) shows, the sum of the velocity head and the pressure head produced in the centrifugal collector with the pitot tube is proportional to the square of the number of rotations per minute.

The data obtained in a series of experiments are plotted in such a graph as Fig. 13 whose horizontal axis is the square of the number of rotations per minute $f^{2}$ and whose vertical axis is the head $H_{c}$ calculated from Eq. (25). The constant $c_{r_{i}}$ in the following equation is derived by applying the least squares method to the plotted data in the graph

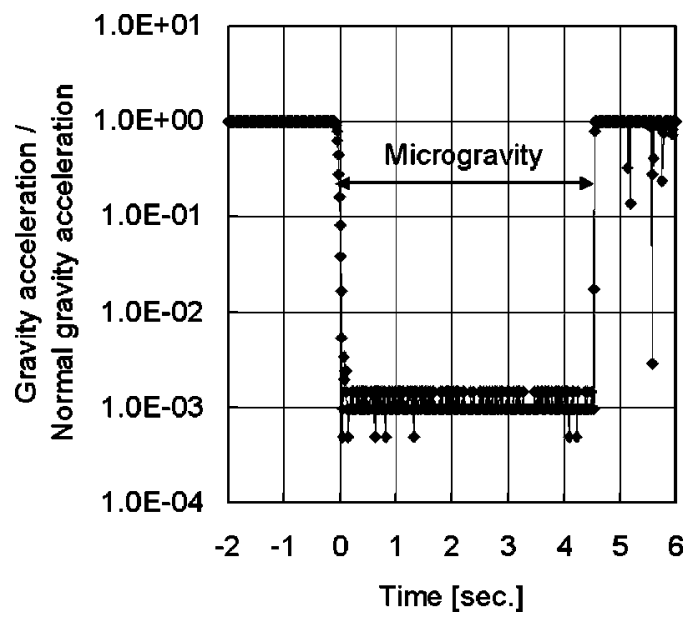

FIG. 11. Ratio of gravity acceleration in an experiment to normal gravity acceleration.

$$
H_{c}=c_{r_{i}} f^{2} .
$$

This equation indicates the pumping performance of the centrifugal collector. Compared with Eqs. (25) and (26), it is found that

$$
c_{r_{i}}=\frac{1}{2 g}\left(2 r_{e}^{2}-r_{i}^{2}\right)\left(2 \pi c \frac{1}{60}\right)^{2} .
$$

The fluid volume in the pitot tube is not replenished during the test, the fluid level $\left(r_{w}-r_{i}\right)$ decreases as $r_{i}$ becomes larger. The working fluid is set at a constant quantity in the centrifugal collector before the experiment starts. Hence, the inner radius at distance $l_{s}$ from the entrance of the pitot tube to the interface between the working fluid and the atmosphere can be identified in each experiment because the quantity of the working fluid in the pitot tube at distance $l_{s}$ is equal in each experiment. It is theoretically predicted that the head obtained by the measurement technique developed in this present work is proportional to the square of the number of rotations per minute. By using $c_{r_{i}}$ derived by applying the least squares method to the plotted data, the constant $c$ can be calculated from Eq. (27). Finally, with substituting the value of the constant $c$ into Eq. (25), the characteristics of the head produced by the centrifugal collector are formulated.

\section{RESULTS AND DISCUSSION}

Figure 11 shows the gravity acceleration relative to normal gravity acceleration in the case that the centrifugal collector was rotated at $187 \mathrm{rpm}$. The experimental support system sent a signal when the microgravity sensor in the experimental support system initially detected a gravity acceleration less than 0.1 times the normal gravity acceleration. In this figure, the horizontal axis denotes time and its origin is the moment at which the signal was sent. As the graph indicates, the gravity acceleration is stable at approximately $1.0 \times 10^{-3}$-fold the normal gravity acceleration from $0.1 \mathrm{~s}$ to $4.5 \mathrm{~s}$. The experiments conducted with other parameters (168 and $223 \mathrm{rpm}$ ) have also been confirmed as being carried out under stable microgravity conditions. 

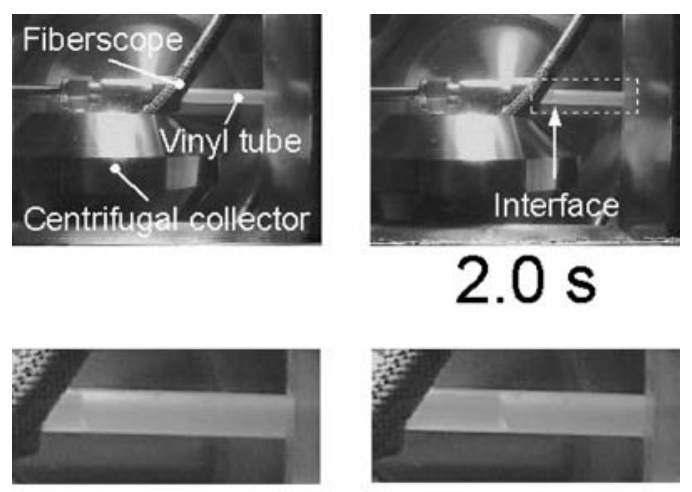

$2.0 \mathrm{~s}$

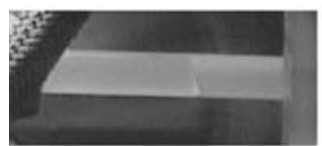

$2.6 \mathrm{~s}$

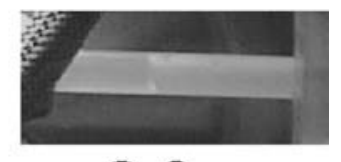

$2.3 \mathrm{~s}$

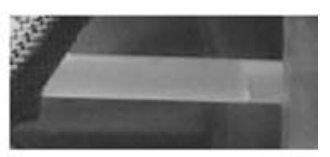

$2.9 \mathrm{~s}$

FIG. 12. Cetrifugal collector and images of the interface at the observational section under normal gravity.

Figure 12 shows images of the centrifugal collector and the interface between the working fluid and the atmosphere in the vinyl tube during an experiment captured on videotape by the $8 \mathrm{~mm}$ video camera. The time after starting a drop is shown under the images. The centrifugal collector was rotated at $187 \mathrm{rpm}$. As the figure shows, the interface between the working fluid and the atmosphere is moving in the vinyl tube. It is notable that the collector for LDRs has the capability to push the working fluid forward. The movement of the working fluid in the vinyl tube is observed to be an almost uniform motion. Analysis of the images has revealed that it takes $1.4 \mathrm{~s}$ for the working fluid to pass through the observational section (between 12.0 and $15.5 \mathrm{~cm}$ from the entrance of the pitot tube). This clarifies that the velocity of the moving interface $u_{s}$ is $0.025 \mathrm{~m} / \mathrm{s}$ in the observational section.

Substituting this value of $u_{s}$ into Eq. (17) as the velocity at the center of the observational section $(0.1375 \mathrm{~m}$ from the entrance of the pitot tube), the head $H_{c}$ produced in the centrifugal collector under microgravity is calculated. The velocity of the interface between the working fluid and the atmosphere in the observational section and the head produced in the centrifugal collector are summarized along with the rotation speed in Table II. Substituting Eq. (2) into the left-hand side of Eq. (1), the following equation is obtained:

$$
H_{c}=\frac{1}{g}\left(\frac{u_{s}^{2}}{2}+\frac{p_{s}}{\rho}+E_{l}\right) .
$$

TABLE II. Surface velocity of working fluid in the vinyl tube and the head produced in the centrifugal collector.

\begin{tabular}{lcc}
\hline \hline $\begin{array}{l}\text { Rotation speed } \\
(\mathrm{rpm})\end{array}$ & $\begin{array}{c}\text { Interface } \\
\text { velocity }(\mathrm{m} / \mathrm{s})\end{array}$ & $\begin{array}{c}\text { Head } H_{c} \\
(\mathrm{~m})\end{array}$ \\
\hline 168 & 0.019 & 0.023 \\
187 & 0.025 & 0.030 \\
223 & 0.034 & 0.041 \\
\hline \hline
\end{tabular}

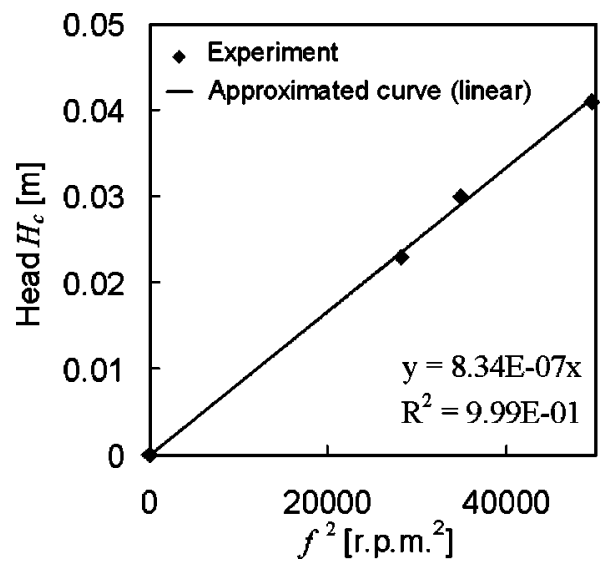

FIG. 13. Head produced in the centrifugal collector vs square of the rotation speed.

In the case that a pressure sensor is mounted downstream of the pitot tube, the velocity of the working fluid at the pressure sensor $u_{s}$ is zero and the total loss of the head $E_{l}$ is derived from Eq. (16) as zero.

Substituting these values of $u_{s}$ and $E_{l}$ into Eq. (28) and then solving for $p_{s}$, the following equation is obtained:

$$
p_{s}=\rho g H_{c} \text {. }
$$

This equation converts the head produced in a centrifugal collector into the pressure measured by a pressure sensor mounted downstream of a pitot tube. The pressure is approximately $390 \mathrm{~Pa}$ in the case of $223 \mathrm{rpm}$. Pressure sensors cannot easily detect this pressure increase in a liquid working fluid with sufficient accuracy.

Figure 13 shows the head $H_{c}$ versus the square of the number of rotations per minute. The line in this figure is the linear approximated curve obtained by using the least squares method. This figure indicates that the head obtained by the measurement technique developed in the present work is proportional to the square of the number of rotations per minute. Since this result is theoretically predicted as shown in Eq. (25), it verifies that the measurement technique developed in the present work can obtain the head produced in the centrifugal collector with reasonable accuracy. Compared with Eq. (26), it follows that

$$
c_{r_{i}}=8.34 \times 10^{-7} \mathrm{~m} / \mathrm{rpm}^{2} \text {. }
$$

When the interface between the working fluid and the atmosphere in the pitot tube is located at $0.1375 \mathrm{~m}$ from the entrance of the pitot tube, the volume of working fluid in the pitot tube is

$$
\pi \times\left(\frac{4.35 \times 10^{-3}}{2}\right)^{2} \times 0.1375=2.04 \times 10^{-6} \mathrm{~m}^{3} .
$$

As the initial volume of the working fluid in the centrifugal collector is $80.0 \times 10^{-6} \mathrm{~m}^{3}$, the volume of the working fluid in the centrifugal collector is $78.0 \times 10^{-6} \mathrm{~m}^{3}$. Using the configuration of the working fluid in the centrifugal collector under microgravity shown in Fig. 4(b) and the dimensions of the centrifugal collector shown in Fig. 7(a), it is calculated that the inner radius of the working fluid in the centrifugal collector $r_{i}$ is $39.5 \times 10^{-3} \mathrm{~m}$. Substituting the values of $c_{r_{i}}$ 


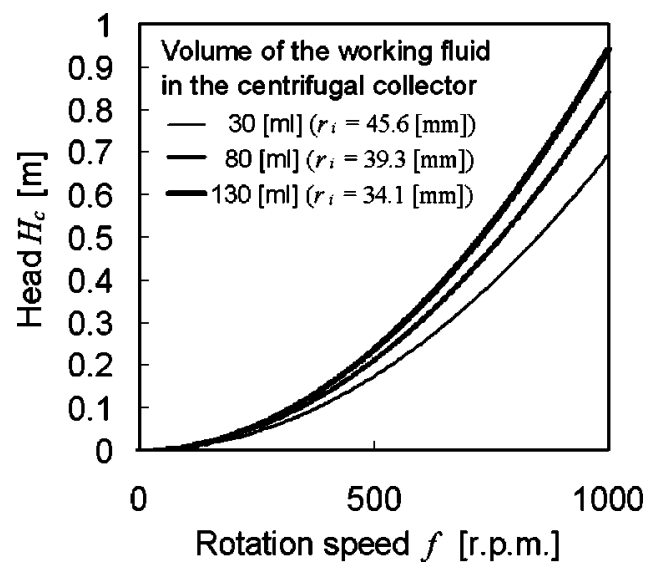

FIG. 14. Head produced in the centrifugal collector vs rotation speed.

and $r_{i}$ into Eq. (27) and solving for $c$, the constant $c$ is derived as $7.00 \times 10^{-1}$. Substituting this value of $c$ into Eq. (25), Eq. (25) becomes the equation for obtaining the head $H_{c}$ produced in the centrifugal collector used in the present work. Figure 14 shows this characteristic for various inner surfaces of the working fluid.

Substituting the values of $c$ and $r_{e}$ into Eq. (23), the velocity at the entrance of the pitot tube $u_{e}$ is obtained: $u_{e}$ $=0.66 \mathrm{~m} / \mathrm{s}$. A comparison between this value of $u_{e}$ and the velocity of the moving interface in the observational section $\left(u_{s}=0.025 \mathrm{~m} / \mathrm{s}\right)$ reveals that the velocity in the observational section decreases to $3.8 \%$ of the velocity at the entrance of the pitot tube. On the other hand, the movement of the interface between the working fluid and the atmosphere in the pitot tube has been observed to be an approximately uniform motion. The remarkable decrease of the velocity of the working fluid in the pitot tube has not been measured in the observational section. Here, the velocity of the working fluid in the pitot tube is examined.

Substituting Eq. (25) into Eq. (2), the following equation is satisfied:

$$
\frac{1}{2 g}\left(2 r_{e}^{2}-r_{i}^{2}\right)\left(2 \pi c \frac{f}{60}\right)^{2}=\frac{1}{g}\left(\frac{u_{s}^{2}}{2}+E_{l}\right)
$$

The loss of energy $E_{l}$ at distance $l_{s}$ from the entrance of the pitot tube is found from Eqs. (3)-(8). The inner radius of the working fluid in the centrifugal collector $r_{i}$ depends on the initial volume of the working fluid in the centrifugal collector and the distance from the entrance of the pitot tube to the interface between the working fluid and the atmosphere. It follows from this that Eq. (30) is the appropriate equation for obtaining the relationship between the velocity $u_{s}$ of the interface and the distance $l_{s}$ from the entrance of the pitot tube to the interface. This relationship is presented in Fig. 15. As the diagram indicates, the velocity of the interface between the working fluid and the atmosphere moving in the pitot tube rapidly decreases after the working fluid enters the pitot tube. Next, the decrease of the velocity becomes gradually gentler, and then the velocity becomes approximately steady. This verifies that the velocity at the entrance of the pitot tube

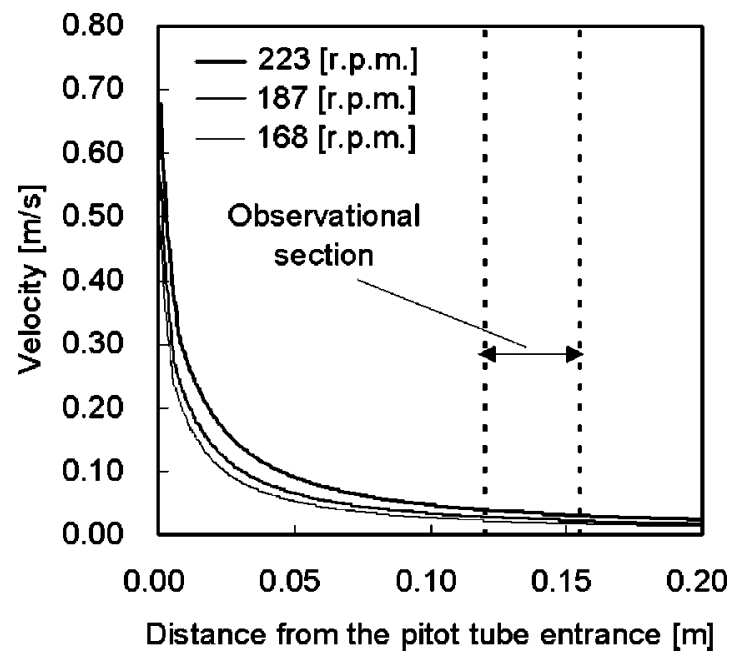

FIG. 15. Velocity of the interface between the working fluid and the atmosphere in the pitot tube.

is significantly different from the velocity in the observational section and the movement of the interface observed in the observational section becomes an almost uniform motion.

\section{DISCUSSION}

In the present work, a technique for measuring the pumping performance under microgravity of a centrifugal collector has been developed and evaluated. The characteristics of the head produced in the centrifugal collector calculated from experimental data agree well with those predicted theoretically from the velocity and the pressure generated by the rotation of the centrifugal collector. It is concluded from this result that the measurement technique can accurately obtain the pumping performance of the centrifugal collector under microgravity. It has also been described that the head produced in a centrifugal collector under microgravity can be predicted from the radius of the entrance of a pitot tube, the inner radius of working fluid in a centrifugal collector, the constant specific to each centrifugal collector and the number of rotations of the centrifugal collector. Although the head cannot be estimated theoretically without obtaining the constant specific to each centrifugal collector by microgravity experiments or other similar means, obtaining the characteristics of the constant specific to each centrifugal collector will allow us to estimate theoretically the head produced in a centrifugal collector under microgravity. It is reasonable to suppose that the constant equals 1 in the case that the circumference of the centrifugal collector is sufficiently large to recover the velocity loss in the wake flow, such as that of the centrifugal collector designed for practical use in space. Nonetheless, it is notable that the technique for measuring the pumping performance described in this article can obtain the head produced under conditions such that the pressure sensors cannot easily detect the pressure increase of the liquid working fluid. This technique is an important contribution to the measurement of the pumping performance under microgravity. 


\section{ACKNOWLEDGMENTS}

The authors are grateful to T. Sasaki for his support in conducting the experiments and creating the figures. This research was carried out as part of the "Ground Research Announcement for Space Utilization" promoted by the Japan Space Forum.

${ }^{1}$ A. T. Mattick and A. Hertzberg, J. Energy 5, 387 (1981).

${ }^{2}$ A. F. Presler, C. E. Coles, P. S. Diem-Kirsop, and K. A. White, Proceedings of the Jt. AIAA/ASME Thermophysics and Heat Transfer Conference, Boston, MA, 2-4 June 1986, ASME 86-HT-15.

${ }^{3}$ A. F. Massardo, L. A. Tagliafico, M. Fossa, and A. Agazzani, J. Propul. Power 13, 560 (1997).

${ }^{4}$ K. A. White, Proceedings of the AIAA 22nd Thermophysics Conference, Honolulu, Hawaii, 8-10 June 1987, AIAA-87-1537.

${ }^{5}$ W. Konopka, V. Calia, and R. Brown, Proceedings of the 20th Intersociety Energy Conversion Engineering Conference, Miami Beach, FL, 18-23 August 1985, Vol. 1, pp. 1430-1438.

${ }^{6}$ S. Hosokawa, M. Kawada, A. Iwasaki, and I. Kudo, J. Jpn. Soc. Aeronautical Space Sciences 41, 385 (1993) (in Japanese).
${ }^{7}$ R. T. Taussig and A. T. Mattick, J. Spacecr. Rockets 23, 10 (1986).

${ }^{8}$ A. White, Aerosp. Am. 32-35 (May 1989).

${ }^{9}$ T. Totani, M. Itami, S. Yabuta, H. Nagata, I. Kudo, A. Iwasaki, and S. Hosokawa, Trans. Jpn. Soc. Mech. Eng., Ser. B 68, 1166 (2002) (in Japanese).

${ }^{10}$ T. Totani, M. Itami, H. Nagata, I. Kudo, A. Iwasaki, and S. Hosokawa, Microgravity Sci. Technol. 13, 42 (2002).

${ }^{11}$ T. Totani, H. Nagara, and I. Kudo, J. Jpn. Soc. Microgravity Application 20, 22 (2003) (in Japanese).

${ }^{12}$ S. Asano and T. Tokuo, Space Forum 6, 411 (2000).

${ }^{13}$ Micro-Gravity Drop Experiment Facility User's Guide (Micro-Gravity Laboratory of Japan Inc., Toki, 2002) (This guide is available from http:// www1.ocn.ne.jp/ mglab/).

${ }^{14}$ S. Sakai and K. Nakatani, Space Forum 6, 403 (2000).

${ }^{15}$ Kiao Ryutai Rikigaku Henshu Iinkai ed., Kiso Ryutai Rikigaku (Sangyou Tosho, Tokyo, 1989), Chap. 6, pp. 45-58 (in Japanese).

${ }^{16}$ F. M. White, Fluid Mechanics, 2nd ed. (McGraw-Hill, New York, 1999), Chap. 6, pp. 325-426.

${ }^{17}$ JSME Mechanical Engineers' Handbook, A. Fundamentals, Vol. A5, Fluid Mechanics (The Japan Society of Mechanical Engineers, 1986), pp. A576-A5-82 (in Japanese). 